\title{
ESTUDOS DE ALGUNS ASPECTOS DA ALIMENTAÇÃO EM ASCIA MONUSTE GODART (LEPIDOPTERA, PIERIDAE)
}

\author{
Maria Cecilia Felipe ${ }^{1,2}$ \\ Fernando Sérgio Zucoloto ${ }^{1}$
}

\begin{abstract}
ESTUDIES ON SOME ASPECTS OF THE ALIMENTATION OF ASCIA MONUSTE GODART (LEPIDOPTERA, PIERIDAE). Six kinds of crucifer (broccoli, cabbage, califlower, kale, mustard and rugula) were tested, as food, for larvae od Ascia monuste. The best results were obtained eith califlower, broccoli, rugula and kale. Four sugars (glucose, fructose, sucrose and maltose) were tested to know their influence on the number of oviposition in female of Ascia monuste. The best results were obtained with fructose, glucose and sucrose. KEY WORDS. Lepidoptera, Pieridae, Ascia monuste, alimentation
\end{abstract}

A performance de qualquer espécie animal depende entre outros fatores, da qualidade e quantidade dos alimentos ingeridos. Nos insetos, a alimentação na fase imatura vai influenciar a variação de peso, o tempo para o desenvolvimento, a sobrevivência, a composição química do corpo e o tamanho do adulto. Na fase adulta, a alimentação é importante para a produção de óvulos, habilidade no cruzamento, sobrevivência e capacidade para dispersão. Em muitas espécies a alimentação na fase imatura influencia vários aspectos da fase adulta; se, por exemplo, a alimentação na fase imatura não for adequada, o adulto poderá emergir, se emergir, retardatariamente, com tamanho reduzido e sem nutrientes estocados, entre outros aspectos. A emergência retardatária poderá desincronizar o adulto em relação à hospedeiros; o tamanho reduzido e a falta de nutrientes estocados poderão prejudicá-los, em relação a produção de feromonas, que afetará a competição por parceiros sexuais, e a produção de óvulos (DADD, 1985; SLANSKY \& SCRIBER, 1985).

Ascia monuste, em sua fase imatura, é uma das maiores consumidoras de crucíferas na Região Neotropical (BRUNINI \& SANTOS, 1975). Trabalhos, feitos com esta espécie, mostram a influência da temperatura no comportamento e desenvolvimento (CORSEUIL \& DA SILVA, 1971; BRUNINI \& SANTOS, 1975; SHIMA \& GOBBI, 1981a) e aspectos da biologia, comparando animais no campo e no laboratório (SHIMA \& GOBBI, 1981b).

$O$ objetivo principal neste trabalho, foi iniciar pesquisas na área de alimentação na espécie $A$. monuste. Pesquisamos dois aspectos: a influência de

1) Departamento de Biologia, Universidade Estadual de São Paulo, Av. Bandeirantes, 3900, 14040-030 Ribeirão Preto, São Paulo, Brasil.

2) Bolsista de Iniciação Científica CNPq. 
diferentes variedades de crucíferas no desenvolvimento do estágio imaturo e a influência de diferentes carboidratos na produção de óvulos, quando fornecidos para as fêmeas adultas.

\section{MATERIAL E MÉTODOS}

As lagartas usadas neste trabalho, foram originárias de ovos coletados em uma horta, perto do Campus da Universidade de São Paulo, em Ribeirão Preto, onde não se aplica agrotóxicos. As variedades de crucíferas testadas (Brassica oleracea) e adquiridas na mesma horta foram: brócoli, couve, couveflor, mostarda, repolho e rúcula. As lagartas recém eclodidas, eram colocadas em caixas de madeira $(8 \times 8 \times 3 \mathrm{~cm})$, tampadas com vidro e forradas com papel jornal. Quando atingiam um tamanho maior, na altura do $7^{\circ}$ dia, eram transferidas para caixas maiores $(19 \times 19 \times 6 \mathrm{~cm})$, nas mesmas condições anteriores. O alimento (folhas novas de diferentes variedades de crucíferas) era renovado diariamente. Cada variedade foi testada 10 vezes, com 15 lagartas por caixa, à uma tempertura de $26^{\circ} \pm 1^{\circ} \mathrm{C}$, umidade relativa ao redor de $70 \%$ e luz fluorescente por $10 \mathrm{~h} / \mathrm{dia}$.

Para se testar o valor nutritivo das diferentes variedades de crucíferas, os seguintes parâmetros foram analisados: peso seco das pupas, tempo para pupação, porcentagem de emergência, comprimento alar das fêmeas, quantidade de oócitos nas fêmeas recém emergidas, digestibilidade aproximada (D.A.), eficiência de conversão do alimento ingerido (E.C.I.) e eficiência de conversão do alimento digerido (E.C.D.). Para a medida do comprimento alar e a contagem dos oócitos, as fêmeas recém emergidas (quatro por experimento, perfazendo um total de 40 para cada variedade testada) eram fixadas em Fixador Dietrich por $48 \mathrm{~h}$, e depois transferidas para uma solução 1:1 de álcool 70\% e glicerina por, no mínimpo, 36h. Então, as fêmeas eram dissecadas, os ovários retirados, corados com carmin acético, montados em lâminas e os oócitos contados. Destas mesmas fêmeas, a asa anterior direita era retirada, e o seu tamanho estimado através da medida entre o início da nervura cubical $(\mathrm{Cu})$ e a bifurcação das radiais 4 e 5 ( $\mathrm{R}_{4}$ e $\left.\mathrm{R}_{5}\right)$. Os valores de $\mathrm{AD}$, $\mathrm{ECI}$ e $\mathrm{ECD}$ foram obtidos segundo SLANSKY \& SCRIBER (1985), a saber:

$$
\mathrm{DA}=\frac{\text { INGESTÃO }- \text { FEZES }}{\text { INGESTÃO }} \cdot 100 \quad \mathrm{ECI}=\frac{\text { BIOMASSA INCORPORADA }}{\text { INGESTÃO }} \cdot 100
$$

$$
\mathrm{ECD}=\frac{\text { BIOMASSA INCORPORADA }}{\text { INGESTÃO }- \text { FEZES }} \cdot 100
$$

A ingestão foi avaliada pela diferença entre o alimento fornecido e as fezes, em períodos de $24 \mathrm{~h}$. Todos os valores foram somados e avaliados em relação à toda fase imatura. Todas as medidas são dadas em peso seco, conseguidas com a seguinte metodologia: as folhas novas eram colhidas três vezes por semana e guardadas em sacos plásticos, no refrigerador. Para a alimentação das lagartas eram usadas sempre as folhas colhidas mais recentemente. Cada 
folha era dividida ao meio, em corte longitudinal; metade era fornecida como alimento, e metade era usada como controle, para sabermos a quantidade de matéria seca contida na folha. $\mathrm{O}$ tamanho e a quantidade de folhas variavam conforme o tamanho das lagartas.

Todo material usado para o cálculo do peso seco (folhas, pupas, exúvias, fezes) era colocado em estufa à $100^{\circ} \mathrm{C}$; pesagens periódicas eram realizadas até a estabilização dos pesos.

Como os cálculos foram feitos para grupos de 15 lagartas, era necessário que todas elas pupassem. Para conseguirmos 10 repetições por variedade de crucífera, com $100 \%$ de pupação, foram feitas tantas repetições quantas necessárias. Este procedimento foi necessário por causa das dificuldades técnicas de se fazer medidas de ingestão e da quantidade de fezes, em lagartas individuais, nos primeiros dias de vida. No caso da biomassa foram computados os pesos das exúvias e das pupas. Para o cálculo do tempo para pupação, porcentagem de emergência, quantidade de oócitos nas fêmeas, mais 10 repetições foram realizadas, para cada variedade de crucífera.

Para estudarmos a influência dos diferentes açúcares sobre a produção de óvulos em $A$. monuste, a seguinte metodologia foi usada: lagartas recém eclodidas eram colocadas para se alimentar em couve, nas mesmas condições já citadas. Quando os adultos emergiam eram colocado em gaiolas $(25 \times 20 \mathrm{x}$ $20 \mathrm{~cm}$ ) de metal revestidas com tule (tecido leve e transparente de algodão), com uma abertura lateral, por onde animais e alimento eram colocados e retirados. Os açúcares testados (glicose, frutose, sacarose e maltose, todos de fabricação da Merck) foram fornecidos à uma concentração $15 \% \mathrm{p} / \mathrm{v}$, e colocados em tampas de plástico com algodão. $\mathrm{O}$ alimento era trocado diariamente; à um grupo controle somente água de torneira era fornecida. Cada grupo era formado de 15 fêmeas e oito machos, e cada açúcar foi testado 10 vezes. Em cada gaiola era colocado um frasco contendo água e uma folha nova de couve, para oviposição. Este material era trocado diariamente. $\mathrm{O}$ número de posturas foi calculado por contagem diária, em cada gaiola. A longevidade das fêmeas foi calculada por observação diária nas gaiolas.

Estes experimentos foram feitos à uma temperatura de $26^{\circ} \mathrm{C} \pm 1^{\circ} \mathrm{C}$ (controlada com aparelho de ar condicionado quente-frio) e $10 \mathrm{~h}$ de luz fluorescente; a umidade relativa não foi controlada. Todos os resultados foram analisados estatisticamente pelo teste de Kruskal Wallis ao nível de significância de 5\% (HOLLANDER \& WOLFE, 1973).

\section{RESULTADOS E DISCUSSÃO}

Os resultados obtidos nos experimentos com lagartas estão sumariados nas tabelas I e II.

Embasados nos resultados obtidos, poderíamos dividir as variedades de crucíferas em dois grupos. Um grupo (A), onde estariam o brócoli, a couve, a couve-flor e a rúcula, que forneceu ótima performance para as lagartas. $\mathrm{O}$ outro grupo (B), onde estariam a mostarda e o repolho, que forneceu uma per- 
Tabela I. Influência de diferentes variedades de crucíferas sobre a porcentagem de emergência, tempo para pupação, comprimento alar em fêmeas e número de oócitos em fêmeas recém emergidas de Ascia monuste. Os resultados representam a média \pm SD de 10 experimentos, com 15 lagartas cada um. Para o comprimento alar e número de oócitos foram usadas 40 fêmeas por variedade de crucífera. Médias seguidas de letras diferentes são diferentes entre si $(P<0,05$, teste de Kruskal-Wallis).

\begin{tabular}{lcccc}
\hline \multicolumn{1}{c}{ Alimento } & $\begin{array}{c}\text { Emergência } \\
(\%)\end{array}$ & $\begin{array}{c}\text { Tempo para } \\
\text { pupação (dias) }\end{array}$ & $\begin{array}{c}\text { Comprimento alar } \\
(\mathrm{cm})\end{array}$ & $\begin{array}{c}\text { № de oócitos } \\
\text { por fêmea }\end{array}$ \\
\hline Brócoli & $96,6 \pm 3,4 \mathrm{a}$ & $21,43 \pm 0,06 \mathrm{a}$ & $2,03 \pm 0,10 \mathrm{a}$ & $505,24 \pm 63,02 \mathrm{a}$ \\
Couve & $90,6 \pm 9,1 \mathrm{a}$ & $20,98 \pm 0,10 \mathrm{a}$ & $1,91 \pm 0,06 \mathrm{a}$ & $571,20 \pm 58,62 \mathrm{a}$ \\
Couve-flor & $98,0 \pm 1,3 \mathrm{a}$ & $22,13 \pm 0,25 \mathrm{a}$ & $1,98 \pm 0,09 \mathrm{a}$ & $548,66 \pm 50,20 \mathrm{a}$ \\
Mostarda & $96,0 \pm 3,2 \mathrm{a}$ & $22,44 \pm 0,83 \mathrm{a}$ & $1,67 \pm 0,08 \mathrm{~b}$ & $327,22 \pm 35,26 \mathrm{~b}$ \\
Repolho & $86,6 \pm 10,4 \mathrm{a}$ & $23,11 \pm 0,09 \mathrm{a}$ & $1,68 \pm 0,10 \mathrm{~b}$ & $306,46 \pm 26,82 \mathrm{~b}$ \\
Rúcula & $85,3 \pm 12,5 \mathrm{a}$ & $22,53 \pm 0,05 \mathrm{a}$ & $1,92 \pm 0,11 \mathrm{a}$ & $434,40 \pm 31,88 \mathrm{c}$ \\
\hline
\end{tabular}

Tabela II. Influência de diferentes variedades de crucíferas sobre o peso seco das pupas, digestibilidade aproximada (DA), eficiência de conversão do alimento ingerido (ECI), eficiência de conversão do alimento assimilado (ECD), ingestão e fezes. Os resultados representam a média \pm SD de 10 experimentos, com 15 lagartas cada um. Médias seguidas de letras diferentes são diferentes entre si $(\mathrm{P}<0,05$, teste de Kruskal Wallis). O peso das pupas inclui as exúvias.

\begin{tabular}{lcccccc}
\hline Alimento & $\begin{array}{c}\text { Peso pupa } \\
(\mathrm{mg})\end{array}$ & $\begin{array}{c}\text { DA } \\
(\%)\end{array}$ & $\begin{array}{c}\text { ECI } \\
(\%)\end{array}$ & $\begin{array}{c}\text { ECD } \\
(\%)\end{array}$ & $\begin{array}{c}\text { Ingestão } \\
(\mathrm{mg})\end{array}$ & $\begin{array}{c}\text { Fezes } \\
(\mathrm{mg})\end{array}$ \\
\hline Brócoli & $66,30 \pm 9,40 \mathrm{a}$ & $46,66 \pm 2,19 \mathrm{a}$ & $17,88 \pm 3,81 \mathrm{a}$ & $38,33 \pm 5,21 \mathrm{a}$ & $370,68 \pm 31,91 \mathrm{a}$ & $197,73 \pm 10,61 \mathrm{a}$ \\
Couve & $63,10 \pm 8,30 \mathrm{a}$ & $45,15 \pm 10,06 \mathrm{a}$ & $15,75 \pm 2,37 \mathrm{a}$ & $34,90 \pm 4,23 \mathrm{a}$ & $400,43 \pm 21,86 \mathrm{a}$ & $219,64 \pm 11,12 \mathrm{a}$ \\
Couve-flor & $65,50 \pm 9,20 \mathrm{a}$ & $38,56 \pm 8,20 \mathrm{a}$ & $17,76 \pm 2,93 \mathrm{a}$ & $46,07 \pm 4,27 \mathrm{~b}$ & $368,70 \pm 14,99 \mathrm{a}$ & $226,53 \pm 16,63 \mathrm{a}$ \\
Mostarda & $53,60 \pm 8,10 \mathrm{~b}$ & $49,14 \pm 9,55 \mathrm{a}$ & $16,49 \pm 3,33 \mathrm{a}$ & $33,57 \pm 4,63 \mathrm{a}$ & $324,87 \pm 10,99 \mathrm{~b}$ & $165,23 \pm 9,31 \mathrm{~b}$ \\
Repolbo & $54,30 \pm 7,60 \mathrm{~b}$ & $47,12 \pm 10,80 \mathrm{a}$ & $13,74 \pm 2,84 \mathrm{~b}$ & $29,17 \pm 2,93 \mathrm{c}$ & $395,06 \pm 19,50 \mathrm{a}$ & $208,91 \pm 11,63 \mathrm{a}$ \\
Rúcula & $67,80 \pm 8,80 \mathrm{a}$ & $42,58 \pm 9,34 \mathrm{a}$ & $18,98 \pm 3,70 \mathrm{a}$ & $44,57 \pm 2,28 \mathrm{~b}$ & $357,21 \pm 28,42 \mathrm{a}$ & $205,11 \pm 13,90 \mathrm{a}$ \\
\hline
\end{tabular}

formance bem inferior para as lagartas.

Considerando todos os parâmetros analisados, teríamos a couve-flor como melhor alimento; esta conclusão é baseada no seguinte: embora a ingestão tenha sido uma das mais baixas e o valor de DA o mais baixo de todos, as lagartas alimentadas com folhas de couve-flor tiveram uma das mais altas porcentagens de emergência, puparam com um dos maiores pesos, consequentemente com as fêmeas adultas tendo um dos maiores tamanhos, e onde se encontrou o segundo maior número de oócitos. O bom valor obtido para o ECI e o ótimo valor obtido para o ECD mostram que as lagartas que se alimentaram de folhas de couve-flor tiveram um excelente aproveitamento do alimento ingerido. As lagartas que se alimentaram de couve conseguiram performance semelhante, ingerindo mais alimento e tendo um maior valor para DA. As lagartas que se alimentavam de couve não tiveram um bom aproveitamento do alimento ingerido, atestado pelos valores obtidos para o ECI e ECD; talvez a alta ingestão e o alto valor para DA sejam adaptações comportamental e fisiológica para compensar o baixo aproveitamento. Para as lagartas alimentadas com brócoli 
vale a mesma análise feita para as lagartas alimentadas com couve. Com exceção para o número de oócitos, as lagartas alimentadas com rúcula tiveram, nos outros parâmetros, performance semelhante às lagartas alimentadas com couve-flor. Entretanto, em uma competição, as fêmeas oriundas de lagartas alimentadas com rúcula deixariam, provavelmemnte, menos descendentes que as outras fêmeas do grupo A. As análises químicas apresentadas em diferentes lugares (AOAC, 1975; BURTON, 1979; FRANCO, 1982) não mostram diferenças acentuadas entre o brócoli, a couve, a couve-flor e a rúcula, entre os nutrientes, incluindo fibra, que é um fator determinante no melhor ou pior aproveitamento do alimento. As análises, entretanto, não incluem quais aminoácidos e em que proporções estão presentes, assim como não incluem análises de aleloquímicos. Estas informações seriam importantes para uma análise mais profunda em relação aos resultados obtidos.

As análises químicas, citadas no parágrafo anterior, mostram para o repolho e a mostarda quantidades inferiores de proteínas e calorias, em relação às outras variedades. Para se ter uma idéia a quantidade de proteínas nas variedades do grupo A varia entre 3,2 à 4,5\%, enquanto é de $2,6 \%$ na mostarda e $1,4 \%$ no repolho; a quantidade de calorias varia de 35 à 44 calorias por $100 \mathrm{~g}$ de alimento nas variedades do grupo A, sendo de 26 na mostarda e 25 no repolho. As quantidades de proteínas e calorias são fundamentais para o desenvolvimento de qualquer ser vivo. Embora estes fatores possam ter sidos decisivos na baixa performance das lagartas alimentadas com mostarda e repolho, outras análises químicas e estudos de comportamento precisam ser realizados para conclusões mais acuradas. Por exemplo, a ingestão nas lagartas alimentadas com mostarda foi a mais baixa de todas e dados, ainda preliminares, mostram uma baixa preferência das lagartas por esta variedade, e também pelo repolho. Estes dados, se confirmados, podem indicar no caso da mostarda uma atratividade baixa, o que levaria à uma baixa ingestão e consequentemente baixa performance, em relação às outras variedades. $\mathrm{O}$ repolho, por outro lado, foi bem ingerido, tendo entretanto, baixos valores para ECI e ECD, o que poderia indicar, realmente, um valor nutritivo inferior. Já no caso da mostarda, o valor do ECI foi bom, mas do ECD foi baixo. Aliás, não houve diferença estatística, entre todos os grupos, para os valores do ECI, com exceção do grupo alimentado com repolho. Isto se deve ao fato de que há uma relação positiva relativa entre a ingestão e a performance com exceção do grupo alimentado com repolho. Os valores do DA para as lagartas alimentadas com mostarda e repolho podem indicar uma tentativa de compensação, para os baixos valores nutritivos destas variedades. Um outro fato importante é que, durante os experimentos, era muito comum observarmos as lagartas que se alimentavam de mostrada e repolho, "vagando" pelas caixas, o que não se observava com frequência nos outros grupos. Talvez, o baixo valor nutritivo da mostarda e do repolho provocassem este tipo de comportamento, ou seja, parece que estão procurando outros alimentos, como já demonstrado em outras espécies (ZUCOLOTO, 1987, 1991; WALDBAUER \& FRIEDMAN, 1991).

Um outro fator importante, que deve ser abordado, é a não diferença 
entre os grupos quanto aos parâmetros: tempo para emergência, porcentagem de emergência e digestibilidade aproximada. Embora a porcentagem de emergência tenha sido alta em todos os grupos, as fêmeas oriundas das lagartas alimentadas com mostarda e repolho apresentaram um número bem inferior de oócitos. A porcentagem de emergência, semelhante em todos grupos, reflete o alto grau de adaptabilidade desta espécie em ralação às crucíferas. É claro que, quantidades diferentes de nutrientes e aleloquímicos, refletem diferenças nas performances dos grupos; entretanto, em nenhum deles, houve resultados decepcionantes; tanto isto é verdade que mantivemos, por mais de 10 gerações, populações alimentadas com todas as variedades. Quando colocamos, aqui, altos e baixos valores nutritivos, são sempre valores relativos abordando as variedades aqui testadas.

Vários fatores afetam o tempo necessário para a pupação, inclusive o alimento (ROCK, 1972). Dependendo da qualidade do alimento, a pupação pode acontecer mais cedo ou mais tarde; existe um peso mínimo que o imaturo deve atingir para pupar (BAKKER, 1961); talvez o peso mínimo seja o que permita a produção do adulto funcional, deixando, desta forma, um certo número de descendentes (SLANSKY \& SCRIBER, 1985). Se a quantidade e a qualidade do alimento, e outros fatores ambientais, estiverem favoráveis, o imaturo poderá exceder este peso mínimo, e alcançar o peso ótimo (SLANSKY \& SCRIBER, 1985). Provavelmente, por isto, o tempo para pupação nas lagartas alimentadas com mostarda e repolho tenha sido semelhante ao dos outros grupos. Poder-se-ia esperar que as lagartas alimentando-se de variedades nutricionalmente inferiores, aumentassem a duração do estágio larval e, em compensação, teriam um tamanho maior ao emergir, com um potencial reprodutivo também maior. Entretanto, pode ser que a "decisão" de puparem mais cedo e com um peso menor seja vantajoso ao garantir adultos funcionais e um certo número de descendentes, evitando um risco maior de predação. O peso das pupas tem influência direta no tamanho dos adultos e, em muitos casos, no número de oócitos.

As diferenças entre os grupos, em relação aos valores do ECD refletem o aproveitamentpo do alimento assimilado, destinados aos gastos metabólicos ou incorporados à biomassa. Se o alimento tem baixo valor nutritivo, seja por deficiência de nutrientes ou aleloquímicos que o animal tem que metabolizar, ele influenciará negativamente o valor de ECD por dois motivos, pelo menos: gasto energético maior na tentativa de compensação do baixo valor nutritivo (aumentando a ingestão, o DA ou mesmo vagando em busca de outros alimentos) e pelo uso que o animal terá que fazer de sua própria biomassa.

Os resultados obtidos com as lagartas de A. monuste, estão de acordo com os resultados obtidos por outros autores, seja com esta mesma espécie, usando a couve como alimento (SHIMA \& GOBBI, 1981a), com Pieris brassicae (SCHOONHOVEN \& MEERMAN, 1978; TIWARI \& KASHYAP, 1988), com $P$. rapae (HOVANITZ, 1969; SLANSKY, 1874), para os parâmetros relativos ao desenvolvimento. Os valores obtidos para DA, ECI e ECD, também estão de acordo com os obtidos para outros lepidópteros, com hábitos alimen- 
tares semelhantes (SLANSKY \& SCRIBER, 1985).

Os resultados obtidos com as fêmeas adultas estão sumariados na tabela III. Os melhores resultados foram obtidos com a frutose, seguidos pela glicose e sacarose. Como houve produção de óvulos nas fêmeas alimentadas só com água, isto indica que uma reserva de nutrientes é trazida da fase imatura. Esta reserva garantiria a sobrevivência e a produção de um certo número de óvulos, mesmo no caso de forte competição ou escassez de alimento. Como estas fêmeas não emergem com óvulos prontos, devem trazer uma certa quantidade de reservas proteicas para terminar o processo. Isto fica evidente quando notamos que alimentando-se de uma fonte de carboidratos, há um aumento dramático na produção de óvulos, o que só pode acontecer tendo-se à disposição proteínas e/ou aminoácidos, que são nutrientes com função primordialmente estrutural (DADD, 1985).

Tabela III. Influência de diferentes carboidratos em solução $15 \%(\mathrm{p} / \mathrm{v})$ sobre a vida média e o número de posturas em A. monuste. Os resultados representam a média $\pm \mathrm{SD}$ de 10 experimentos, com 15 fêmeas e oito machos por grupo. Médias seguidas de letras diferentes são diferentes entre si $(\mathrm{P}<0,05$, teste de Kruskal-Wallis).

\begin{tabular}{lcc}
\hline \multicolumn{1}{c}{ Alimento } & Vida média & Número de posturas \\
\hline Água & $5,23 \pm 2,07 \mathrm{a}$ & $26,27 \pm 5,57 \mathrm{a}$ \\
Glicose & $7,57 \pm 2,31 \mathrm{~b}$ & $83,51 \pm 10,24 \mathrm{~b}$ \\
Frutose & $7,12 \pm 3,01 \mathrm{~b}$ & $151,70 \pm 19,67 \mathrm{c}$ \\
Sacarose & $7,81 \pm 2,43 \mathrm{~b}$ & $71,20 \pm 9,36 \mathrm{~b}$ \\
Maltose & $3,23 \pm 2,07 \mathrm{c}$ & $4,29 \pm 1,03 \mathrm{~d}$ \\
\hline
\end{tabular}

Os baixos resultados proporcionados pela maltose (inferiores, inclusive, aos obtidos com o grupo controle alimentado só com água), indicam que estas fêmeas não devem possuir enzimas para digerir este dissacarídeo. Embora não tenhamos medido a ingestão dos carboidratos, observações feitas mostraram que tanto fêmeas como machos ingeriam da solução de maltose, com a mesma frequência que os outros grupos ingeriam os carboidratos correspondentes. A glicose, produto final da digestão da maltose, proporcionou bons resultados descartando, portanto, problemas metabólicos. Em geral a enzima que digere maltose digere, também, a sacarose, $\alpha$-glucosidase; entretanto, outra enzima, $\alpha$-fructosidase digere a sacarose, mas não a maltose (BURSELL, 1970). Pode ser, então, que as fêmeas adultas de $A$. monuste possuam $\alpha$-fructosidase, que digeriria a sacarose, e não possua $\alpha$-glucosidase, que digeririra a maltose. Resultados semelhantes foram encontrados em fêmeas adultas de Ceratitis capitata que se alimentam, entre outras coisas, de nectar, como as fêmeas de $A$. monuste (ZUCOLOTO, no prelo). Em uma outra espécie, também de Lepidoptera, Erinnyis ello, análises enzimáticas mostraram resultados que apoiaram esta hipótese (SANTOS \& TERRA, 1986). Não digerindo a maltose, as fêmeas podem ter tido a vida média reduzida, pelo acúmulo do açúcar no trato digestivo, causando problemas osmóticos. 
Como as fêmeas adultas desta espécie se alimentam basicamente de néctares, substâncias ricas em açúcares, principalmente glicose, frutose e sacarose, mas pobres em maltose (GOTHILF et al., 1971; WILLS et al., 1981; KEVAN et al., 1983; DAFNI et al., 1988), os resultados obtidos seriam os esperados. A pergunta que fica é: será que a não utilização de maltose pelas fêmeas adultas que se alimentam de nectar, seria um fenômeno generalizado?

O valor nutritivo de um carboidrato depende de três fatores: 1) o seu grau de atratividade (o que influenciaria na quantidade ingerida); 2) a presença de enzimas digestivas para reduzir os carboidratos complexos aos seus monomeros e 3) a utilização metabólica dos monossacarídeos. De uma maneira geral os carboidratos melhores utilizados pelos insetos, são aqueles que se encontram em seus alimentos naturais (DADD, 1985). Os dados, aqui relatados, confirmam esta hipótese.

Vários estudos mostram a influência dos açúcares sobre vários aspectos dos lepidópteros, inclusive sugerindo que a fonte destes açúcares pode ter importante papel na seleção de habitat. Alguns resultados da literatura são semelhantes aos obtidos neste experimento (ADLER, 1989).

\section{REFERÊNCIAS BIBLIOGRÁFICAS}

ADLER, P.H. 1989. Sugar feeding of the adult corn earworm in the laboratory. J. Econ. Entomol. 82 (5): 1344-1349.

AOAC. 1975. Official Analytical Chemists Association. $12^{\text {th }}$ edition. Washington, $927 \mathrm{p}$.

BAKKER, K. 1961. An analysis of factors which determine success in competition for food among larvae of Drosophila melanogaster. Arch. Neerl. Zool. 14: 200-281.

BRUNINI, O. \& J.M. SANTOS. 1975. Comportamento de Ascia monuste orseis em função da temperatura do ar. Ciênc. Cult. 28 (6): 681-683.

BURSELL, E. 1970. An Introduction To Insect Physiology. Academic Press, London, 276p.

BURTON, B. 1979. Nutrição humana. São Paulo, McGraw-Hill, 606p.

CORSEUIL, E. \& T.L. DA SILVA. 1971. Efeito da baixa temperatura no desenvolvimento de Ascia monuste orseis. Agron. sulriog. 7 (2): 191-196.

DADD, R.H. 1985. Nutrition: Organisms, p.313-389. In: G.A. KERKUT \& L.I. GILBERT (ed.) Comprehensive Insect Physiology, Biochemiostry and Pharmacology IV. Oxford, Pergamon Press.

DAFNI, H.; Y. LENSKY \& A. FAHN. 1988. Flower and nectar characteristics of nine species of Labiatae and their influence on honeybees visits. J. Apic. Res. 27 (2): 103-114.

FRANCO, G. 1982. Nutrição. Livraria Atheneu, $6^{a}$ ed., 227p.

GOTHILF, S.R.; R. GALUN \& M. BAN-ZEER. 1971. Teste reception in the Mediterranean fruit-fly: electrophysiological and behavioural studies. J. Insect Physiol. 17: 1371-1384. 
HOLLANDER, M. \& D.A. WOLFE. 1973. Non-Parametric Statistical Methods. New York, John Wiley and Sons, 503p.

HOVANITZ, W. 1969. Inherited and/or conditioned changes in host-plant preference in Pieris. Ent. exp. appl. 12: 729-735.

KEVAN, P.G.; S. STHELENS \& I. BAKER. 1983. Honey bees feeding from honeydew exudate of diseased Gambel's oak in Colorado. J. Apic. Res. 22 (1): 53-56.

ROCK, G.C. 1972. Optimal proportions of dietary amino acids. In: Insect and Mite Nutrition. Amsterdam, North-Holland Publish, 183-197.

SANTOS, C.D. \& W.R. TERRA. 1986. Midgut $\alpha$-glucosidase and $\beta$-fructosidase from Erinnyis ello larvae and imagos. Physical and Kinetic properties. Insect Biochem. 16: 819-824.

SCHOONHOVEN, L.M. \& J. MEERMAN. 1978. Metabolic cost of changes in diet and neutralization of allelochemics. Ent. exp. appl. 24: 689-693.

SHIMA, S.N. \& N. GOBBI. 1981a. Preliminary studies of some factors that influence mating behaviour of Ascia monuste orseis under laboratory conditions. Rev. Brasil. Biol. 41 (3): 663-666.

1981b. Estudo de alguns aspectos da biologia de Ascia monuste orseis em condições de laboratório e campo. Rev. bras. Ent. 25 (4): 313-319.

SLANSKY, F. 1974. Energetic and nutritional interactions between larvae of the imported cabbage butterfly, Pieris rapae $\mathrm{L}$. and cruciferous plants. Tese de Doutorado, não publicada, Cornell University, Ithaca, N.Y. Apud: Slansky, F. \& J.M. Scriber. 1985. Food consumption and utilization, p.87-163. In: G.A. KERKUT \& L.I. GILBERT (ed.). Comprehensive Insect Physiology, Biochemistry and Pharmacology. Vol. III, Oxford, Pergamon Press.

SLANSKY, F. \& SCRIBER, J.M. 1985. Food consumption and utilization, p. 87-163. In: G.A. KERKUT \& L.I. GILBERT (ed.). Comprehensive Insect Physi- ology, Biochemistry and Pharmacology. Vol. III, Oxford, Pergamon Press.

TIWARI, S.N. \& N.P. KASHYAP. 1988. Feeding and oviposition preference of cabbage butterfly Pieris brassicae. Bull. Annls. Soc. r. belge Ent. 124: 131-135.

WALDBAUER, G.P. \& FRIEDMAN, S. 1991. Self-selection of optimal diets by insects. Ann. Rev. Entomol. 36: 43-63.

WILLS, R.B.H., LEE, T.H., GRAHAM, D. MCGLASSON, W.B. \& HALL, E.G. 1981. Postharvest: An Introduction to the Physiology and Handling of Fruit and Vegetables. Westport, Connecticut, The AVI Publishing Company Inc., 166p.

ZUCOLOTO, F.S. 1987. Feeding habits of Ceratitis capitata: Can larvae recognize a nutritionally effective diet? J. Insect physiol. 33: 349-354.

. 1991. Effects of flavour and nutritional value on diet selection by Ceratitis capitata larvae. J. Insect. Physiol. 37: 21-25.

. (IN PRESS). Egg production by females of Ceratitis capitata fed with different carbohydrates. Rev. bras. Ent.

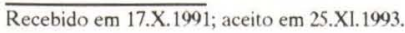

